\title{
Modeling of Roller Bearings
}

\author{
Dipl.-Ing. Tobias Weiser ${ }^{1}$ Univ.-Prof. Dr.-Ing. Burkhard Corves ${ }^{2}$ \\ ${ }^{1}$ KUKA Robotics GmbH, Germany, Tobias . Weiser@kuka.com \\ ${ }^{2}$ Department of Mechanism Theory and Dynamics of Machines, RWTH Aachen, Germany, \\ corveseigm.rwth-aachen. de
}

\begin{abstract}
Modeling of multi-body system mechanics plays a central role in the design of mechatronic systems. Roller bearings contribute stiffness and damping to the system dynamics of a mechatronic system. This article shows the stiffness modeling of selected types of roller bearings. The kinematics of deformation of a roller bearing are shown. Based on the principle of Hertz'ian contact stress the elastic forces and torques are calculated. These forces are considered and implemented in the MultiBody Library.

Keywords: Bearing Stiffness, Bearing Modelling, MultiBody Library
\end{abstract}

\section{Introduction}

This document discusses the modeling of stiffness for various types of rolling-contact bearings for use in the simulation of multi-body systems. In addition to this macroscopic perspective, the modeling of stiffness is used for rotordynamics. Beyond this, research in bearing modeling deals with the effects on structure-borne noise. The study (Ghalamchi et al., 2013) puts forward a simple model based on Hertz'ian contact stress to calculate rotordynamics for barrel roller bearings. A second application for bearing modeling is damage diagnostics and the identification of the causes of bearing damage. In the publication (Tadina and Boltežar, 2011), the system-dynamic effects of damage to the balls and running surfaces of ball bearings are analyzed. The inner ring, the outer ring and the rolling elements are modeled as rigid bodies. The balls are elastically connected to the inner ring and the outer ring. To test design measures to improve the contact pattern of the bearing and its rolling elements, system-dynamic investigations are carried out. To calculate the optimal profiles for cylindrical roller bearings, the rollers are discretized in (Qian and Jacobs, 2014) using the slice model, and the stiffness is modeled as a Hertz'ian contact.

The scope of this paper is to create a model for the stiffness of selected types of single and double row roller bearings. It will be used for modeling mechanisms like a robot arm. The target is to simulate macroscopic system dynamics of a mechanism.

Modelica provides a powerful library for simulating multi-body systems like mechanisms - the MultiBody library. Therefore this approach extends the range of this library. An internal bearing analysis is out of scope of this model. Effects considering the rolling elements e.g. mass effects are neglected.

\section{Single-row bearings}

For the modeling of roller bearings or ball bearings, the elastic forces and torques are determined for both types using the Hertz'ian contact stress.
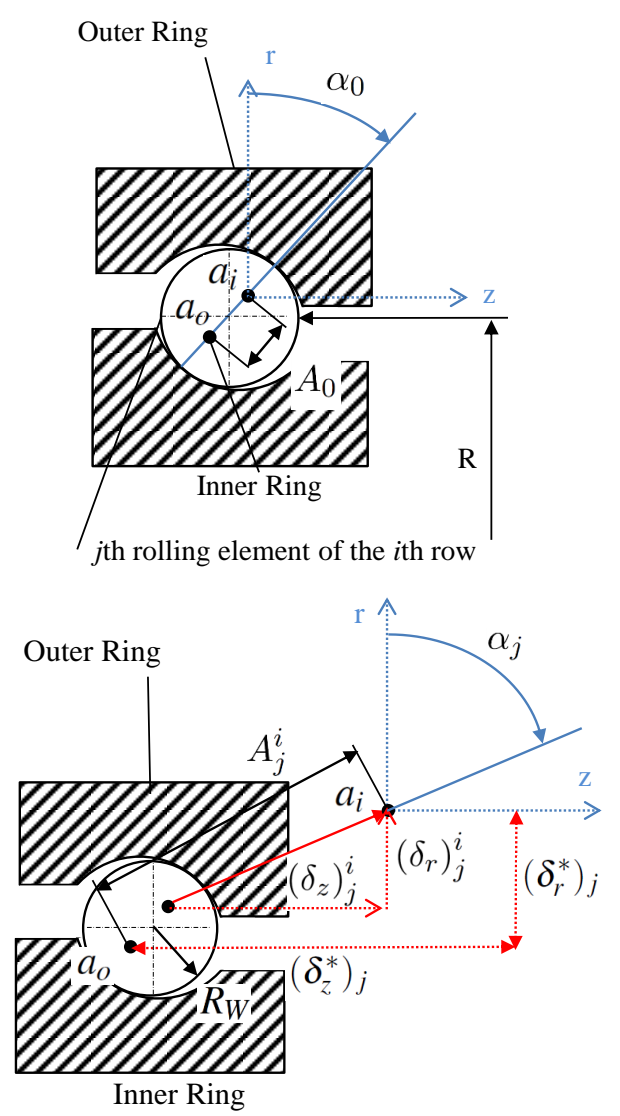

Figure 1. Deformations of a bearing

For simulating the stiffness of a bearing in Modelica the forces and torques in a bearing have to be derived.

First the change in angular position of the rolling element $j$ when rotating the bearing about the $\mathrm{Z}$-axis with the angle $\varphi$ is calculated using the bearing geometry of the pitch radius of the rolling elements $R$ and the rolling element radius $R_{W}$. 


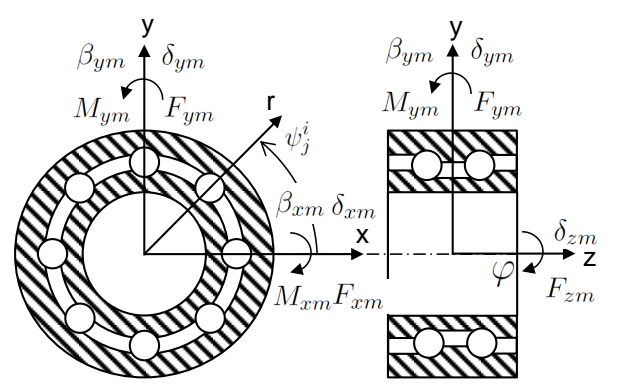

Figure 2. Coordinate system of a double row bearing

$$
\Delta \psi_{j}=\left\{\begin{array}{l}
\frac{R-R_{W} \cos \left(\alpha_{0}\right)}{R} \cdot \varphi, \text { fixed outer ring } \\
\frac{R+R_{W} \cos \left(\alpha_{0}\right)}{R} \cdot \varphi, \text { fixed inner ring }
\end{array}\right.
$$

We simplify in the next equation the angle $\psi_{j}$ of the roller position of the $j t h$ roller according to the $\mathrm{X}$-axis to the angle $\widetilde{\psi}_{j}$ :

$$
\widetilde{\psi}_{j}=\psi_{j}+\Delta \psi_{j}
$$

For every rolling element $j$, the axial $\delta_{z j}$ and radial deformation $\delta_{r j}$ is calculated from the translational deformations $\delta_{x m}, \delta_{y m}, \delta_{z m}$ and the angular deformations $\beta_{x m}, \beta_{y m}$ of the bearing (Fig. 2) with the pitch radius $R$ and the bearing clearance $r_{L}$.

$$
\begin{aligned}
\delta_{z j}= & \delta_{z m}+R\left(\beta_{x m} \sin \left(\widetilde{\psi}_{j}\right)\right. \\
& \left.-\beta_{y m} \cos \left(\widetilde{\Psi}_{j}\right)\right) \\
\delta_{r j}= & \delta_{x m} \cos \left(\widetilde{\Psi}_{j}\right)+ \\
& \delta_{y m} \sin \left(\widetilde{\psi}_{j}\right)-r_{L}
\end{aligned}
$$

The contact angle under load $\alpha_{j}$ of the rolling element $\mathrm{j}$ can be calculated with the contact angle of the bearing $\alpha_{0}$, the net effective radial $\left(\delta_{r}^{*}\right)_{j}$ and axial $\left(\delta_{z}^{*}\right)_{j}$ displacement and the relative distance $A_{0}$ between the raceway groove curvature centers of the inner $a_{i}$ and outer $a_{o}$ bearing ring in case of no load:

$$
\begin{aligned}
\left(\delta_{z}^{*}\right)_{j} & =A_{0} \sin \left(\alpha_{0}\right)+\delta_{z j} \\
\left(\delta_{r}^{*}\right)_{j} & =A_{0} \cos \left(\alpha_{0}\right)+\delta_{r j} \\
\tan \left(\alpha_{j}\right) & =\frac{\left(\delta_{z}^{*}\right)_{j}}{\left(\delta_{r}^{*}\right)_{j}}
\end{aligned}
$$

Then we obtain the distance between the raceway groove curvature centers under load $A_{j}$ :

$$
A_{j}=\sqrt{\left(\delta_{z}^{*}\right)_{j}^{2}+\left(\delta_{r}^{*}\right)_{j}^{2}}
$$

Depending on the type of the rolling element $W$, the total elastic deformation is $\delta_{W}\left(\psi_{j}\right),(W=B:$ ball, $R$ : roller).

$$
\begin{aligned}
\text { Ball : } & \\
\delta_{B, j} & =\left\{\begin{array}{c}
A_{j}-A_{0}, \delta_{B j}>0 \\
0, \delta_{B j} \leq 0
\end{array}\right.
\end{aligned}
$$

Roller :

$$
\delta_{R, j}=\left\{\begin{array}{c}
\delta_{r j} \cos \left(\alpha_{j}\right)+\delta_{z j} \sin \left(\alpha_{j}\right), \delta_{R j}>0 \\
0, \delta_{R j} \leq 0
\end{array}\right.
$$

The load $Q_{j}$ on each rolling element $j$ depends on the type of roller bearing $W$ and is calculated with the Hertz'ian exponent $n$. For elliptical Hertz'ian contact (ball - elastic half-space) we assume $n=3 / 2$. For a rectangular contact (cylinder - elastic half-space) we assume $n=10 / 9$. Depending on the geometry and the material properties of the roller we denote the load $Q_{j}$ on each rolling element $j$ with the Hertz'ian stiffness constant $K_{n}$ for ball $(B)$ and roller bearings $(R)$ :

$$
Q_{j}=K_{n} \cdot \delta_{W}\left(\widetilde{\psi_{j}}\right)^{n}
$$

Then we yield the forces and torques on a bearing with $Z$ rolling elements and $\widetilde{\psi}_{j}$ as the angular position of each rolling element (Lim and Singh, 1990a,b; Gunduz, 2012).

$$
\left[\begin{array}{c}
F_{x b m} \\
F_{y b m} \\
F_{z b m} \\
M_{x b m} \\
M_{y b m} \\
M_{z b m}
\end{array}\right]=\sum_{j=1}^{Z} Q_{j}\left[\begin{array}{c}
\cos \left(\alpha_{j}\right) \cos \left(\widetilde{\psi}_{j}\right) \\
\cos \left(\alpha_{j}\right) \sin \left(\widetilde{\psi}_{j}\right) \\
\sin \left(\alpha_{j}\right) \\
R \sin \left(\alpha_{j}\right) \sin \left(\widetilde{\psi}_{j}\right) \\
-R \sin \left(\alpha_{j}\right) \cos \left(\widetilde{\psi}_{j}\right) \\
0
\end{array}\right]
$$

For roller bearings we obtain with $\alpha_{j}=\alpha_{0}$ (Lim and Singh, 1990a) the forces and torques:

$$
\begin{aligned}
{\left[\begin{array}{c}
F_{x b m} \\
F_{y b m} \\
F_{z b m} \\
M_{x b m} \\
M_{y b m} \\
M_{z b m}
\end{array}\right]=} & K_{n} \sum_{j=1}^{Z}\left(\delta_{R, j} \cos \left(\alpha_{0}\right)\right)^{n} \\
& {\left[\begin{array}{c}
\cos \left(\alpha_{0}\right) \cos \left(\widetilde{\psi}_{j}\right) \\
\cos \left(\alpha_{0}\right) \sin \left(\widetilde{\psi}_{j}\right) \\
\sin \left(\alpha_{0}\right) \\
R \sin \left(\alpha_{0}\right) \sin \left(\widetilde{\psi}_{j}\right) \\
-R \sin \left(\alpha_{0}\right) \cos \left(\widetilde{\psi}_{j}\right) \\
0
\end{array}\right] }
\end{aligned}
$$

The rotation about the $Z$-Axis is free because the torque about the $Z$-axis $M_{z b m}$ in equations 12 and 13 is zero.

\section{Double-row bearings}

Besides single-row the force and torque balances for double-row ball and roller bearings are determined in this section. Three possible configurations for the double row bearings exist (Figure 3). The bearing arrangement coefficent $c_{3}$ considers these configurations. The calculations of the radial $\left(\delta_{r}\right)_{j}^{i}$ and axial displacement $\left(\delta_{z}\right)_{j}^{i}$ of 

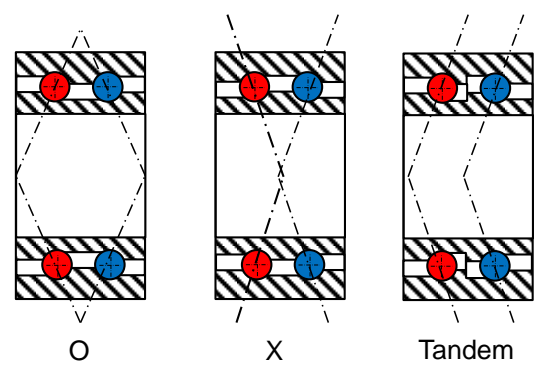

Figure 3. Bearing Arrangements (Matek et al., 2013) (Gunduz, 2012)

each rolling element $j$ in row $i$ (equations 3,4 ) have to be extended by the kinematics of the double row bearing with the row coefficent $c_{1}$, the bearing clearance $r_{L}$, the distance between the two bearing rows $e$, the translational deformations $\delta_{x m}, \delta_{y m}, \delta_{z m}$, the angular deformations $\beta_{x m}, \beta_{y m}$ of the bearing and the angular position $\widetilde{\psi}_{j}^{i}$ of each rolling element.

$$
\begin{aligned}
& c_{1}=\left\{\begin{array}{cl}
1, & \text { for } \mathrm{i}=1, \text { left row } \\
-1, & \text { for } \mathrm{i}=2, \text { right row }
\end{array}\right. \\
& \left(\delta_{z}\right)_{j}^{i}=\left[\delta_{x m}+c_{1} \beta_{y m} e\right] \cos \left(\widetilde{\psi}_{j}^{i}\right)+ \\
& {\left[\delta_{y m}-c_{1} \beta_{x m} e\right] \sin \left(\widetilde{\psi}_{j}^{i}\right)-r_{L}} \\
& \left(\delta_{r}\right)_{j}^{i}=\delta_{z m}+R\left[\beta_{x m} \sin \left(\widetilde{\psi}_{j}^{i}\right)-\beta_{y m} \cos \left(\widetilde{\psi}_{j}^{i}\right)\right]
\end{aligned}
$$

Next the equations of the net effective radial $\left(\delta_{r}^{*}\right)_{j}^{i}$ and axial $\left(\delta_{z}^{*}\right)_{j}^{i}$ displacement of equation 5 and 6 are extended for the double row bearings. With the bearing arrangement coefficent $c_{3}$ (Figure 3) we calculate the net radial $\left(\delta_{r}^{*}\right)_{j}^{i}$ and axial $\left(\delta_{z}^{*}\right)_{j}^{i}$ effective displacements and the loaded distance $A_{j}^{i}$ between the raceway groove curvature centers of the inner $a_{i}$ and outer $a_{o}$ bearing ring of the rolling element $j$ in the row $i$.

$$
\begin{aligned}
& c_{3}=\left\{\begin{array}{l}
{[\text { left row, right row }], \text { arrangement }} \\
{[1,-1], \quad \text { Back-To-Back, O }} \\
{[-1,1], \quad \text { Face-To-Face, X }} \\
{[1,1], \quad \text { Tandem }}
\end{array}\right. \\
&\left(\delta_{r}^{*}\right)_{j}^{i}=A_{0} \cos \left(\alpha_{0}\right)+\delta_{r j}^{i} \\
&\left(\delta_{z}^{*}\right)_{j}^{i}=\delta_{z j}^{i}+c_{3}\left(A_{0} \sin \left(\alpha_{0}\right)+\delta_{z 0}^{i}\right) \\
& A_{j}^{i}=\left(\sqrt{\left.\left(\delta_{z}^{*}\right)_{j}^{i}\right)^{2}+\left(\left(\delta_{r}^{*}\right)_{j}^{i}\right)^{2}}\right.
\end{aligned}
$$

In general, the following relation applies to the forces $F_{x b m}, F_{y b m}, F_{z b m}$ and torques $M_{x b m}, M_{y b m}, M_{z b m}$ on the double-row ball bearing. For simplification we introduce the parameter $R^{*}$.

$$
\begin{aligned}
{\left[\begin{array}{c}
F_{x b m} \\
F_{y b m} \\
F_{z b m} \\
M_{x b m} \\
M_{y b m} \\
M_{z b m}
\end{array}\right] } & =\sum_{i=1}^{2} \sum_{j=1}^{Z} Q_{j}\left[\begin{array}{c}
\cos \left(\alpha_{j}^{i}\right) \cos \left(\widetilde{\boldsymbol{\psi}}_{j}^{i}\right) \\
\cos \left(\alpha_{j}^{i}\right) \sin \left(\widetilde{\boldsymbol{\psi}}_{j}^{i}\right) \\
\sin \left(\alpha_{j}^{i}\right) \\
R^{*} \sin \left(\widetilde{\boldsymbol{\psi}}_{j}^{i}\right) \\
-R^{*} \cos \left(\widetilde{\boldsymbol{\psi}}_{j}^{i}\right) \\
0
\end{array}\right] \\
R^{*} & =R \sin \left(\alpha_{j}^{i}\right)-c_{1} e \cos \left(\alpha_{j}^{i}\right)
\end{aligned}
$$

For bearings with rollers as rolling elements, the angular offset $\alpha_{i}^{j}$ assumed to be zero. Therefore, the following applies:

$$
\alpha_{j}^{i}=\alpha_{0}
$$

The equations for the forces $F_{x b m}, F_{y b m}, F_{z b m}$ and torques $M_{x b m}, M_{y b m}, M_{z b m}$ are thus as follows for each rolling element $j$ in row $i$ introducing the parameter $\widetilde{R}$ for simplification.

$$
\begin{aligned}
{\left[\begin{array}{c}
F_{x b m} \\
F_{y b m} \\
F_{z b m} \\
M_{x b m} \\
M_{y b m} \\
M_{z b m}
\end{array}\right] } & =\sum_{i=1}^{2} \sum_{j=1}^{Z} Q_{j}\left[\begin{array}{c}
\cos \left(\alpha_{0}\right) \cos \left(\widetilde{\psi}_{j}^{i}\right) \\
\cos \left(\alpha_{0}\right) \sin \left(\widetilde{\psi}_{j}^{i}\right) \\
\sin \left(\alpha_{0}\right) \\
\widetilde{R} \sin \left(\widetilde{\psi}_{j}^{i}\right) \\
-\widetilde{R} \cos \left(\widetilde{\psi}_{j}^{i}\right) \\
0
\end{array}\right] \\
\widetilde{R} & =R \sin \left(\alpha_{0}\right)-c_{1} e \cos \left(\alpha_{0}\right)
\end{aligned}
$$

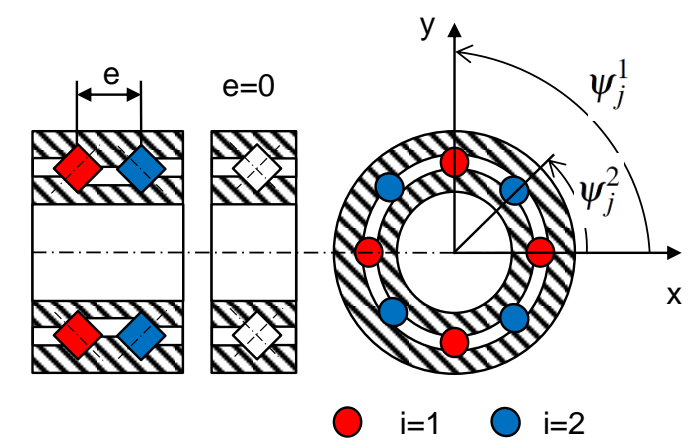

Figure 4. Conversion of a double row roller bearing to a cross roller bearing

A cross-roller bearing is modeled as a double-row bearing for which the distance $e$ between both bearing rows is zero (see Fig. 4). For each row the correct angle offset $\widetilde{\psi}_{j}^{i}$ for each rolling element has to be considered.

\section{Implementation}

The integration is carried out in the MultiBody library. In the preceding chapters, the relationship of the forces and torques on a roller bearing has been described. The motion equations and the elastic forces and torques of the bearing are determined using Lagrange's equation of the 
second kind. Here $T$ is the kinetic energy, $\mathbf{Q}$ are the generalized forces (Hardtke et al., 1997). The generalized forces $\mathbf{Q}$ consist of conservative $\mathbf{Q}_{k}$ and non-conservative forces $\mathbf{Q}_{n}$. In this model non-conservative forces don't exist. With the vector of generalized coordinates $\mathbf{q}$ and the potential energy $U(\mathbf{q})$ we denote:

$$
\begin{aligned}
\frac{d}{d t}\left(\frac{\partial T}{\partial \dot{q}_{i}}\right)-\frac{\partial T}{\partial q_{i}} & =\mathbf{Q} \\
\mathbf{Q} & =\mathbf{Q}_{k}+\mathbf{Q}_{n} \\
\mathbf{Q}_{n} & =\mathbf{0} \\
\mathbf{Q}_{k}(\mathbf{q}) & =-\frac{\partial U(\mathbf{q})}{\partial \mathbf{q}}
\end{aligned}
$$

The vector of generalized coordinates $\mathbf{q}$ are the deformations on the bearing and we yield the vector of the forces on a rolling element $\mathbf{F}(\mathbf{q})$ with the auxiliary parameters for coding $\mathbf{F}_{\text {Bearing }}, \mathbf{M}_{\text {Bearing }}$ :

$$
\begin{aligned}
\mathbf{q} & =\left[\boldsymbol{\delta}_{x m}, \boldsymbol{\delta}_{y m}, \boldsymbol{\delta}_{z m}, \beta_{x m}, \boldsymbol{\beta}_{y m}\right]^{T} \\
\mathbf{F}(\mathbf{q}) & =\left[\mathbf{F}_{\text {Bearing }}, \mathbf{M}_{\text {Bearing }}\right]^{T} \\
\mathbf{F}_{\text {Bearing }} & =\left[F_{x b m}, F_{y b m}, F_{z b m}\right]^{T} \\
\mathbf{M}_{\text {Bearing }} & =\left[M_{x b m}, M_{y b m}, 0\right]^{T}
\end{aligned}
$$

The force and torque relationships for single-row and double-row bearings are described in chapter 2 and chapter 3 , respectively. For calculation of the potential energy $U(\mathbf{q})$, the following applies in general for the energy over the force $\mathbf{F}(\mathbf{q})$ with the vector of generalized deformations $\mathbf{q}$ and finally we yield for the conservative force $\mathbf{Q}_{k}$ :

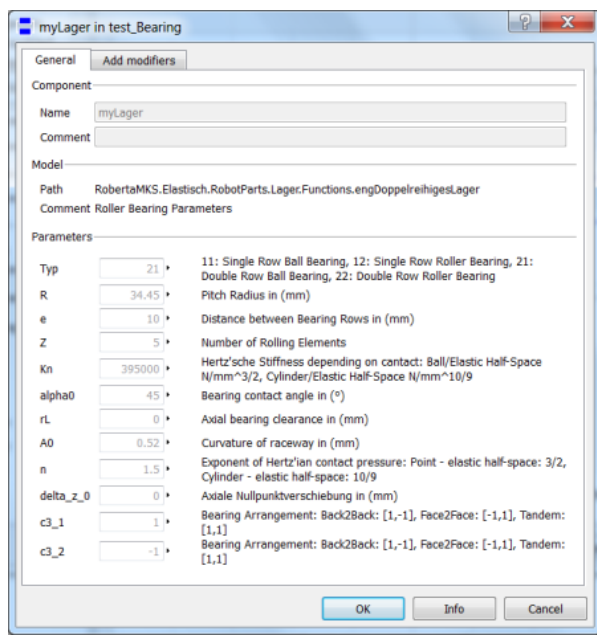

Figure 5. Input parameter mask in Dymola

$$
\begin{aligned}
\frac{\partial U}{\partial q_{i}} & =\frac{\partial}{\partial q_{i}}\left(\int_{0}^{q} \mathbf{F}\left(q_{i}\right) \mathbf{d} q_{i}\right) \\
\frac{\partial U}{\partial q_{i}} & =\mathbf{F}\left(q_{i}\right) \\
\mathbf{Q}_{k} & =-\mathbf{F}(\mathbf{q})
\end{aligned}
$$

The equations 35 and 36 show that the relationships of the forces and torques can be directly incorporated into the equations of motion.

The force and torque equilibrium then is:

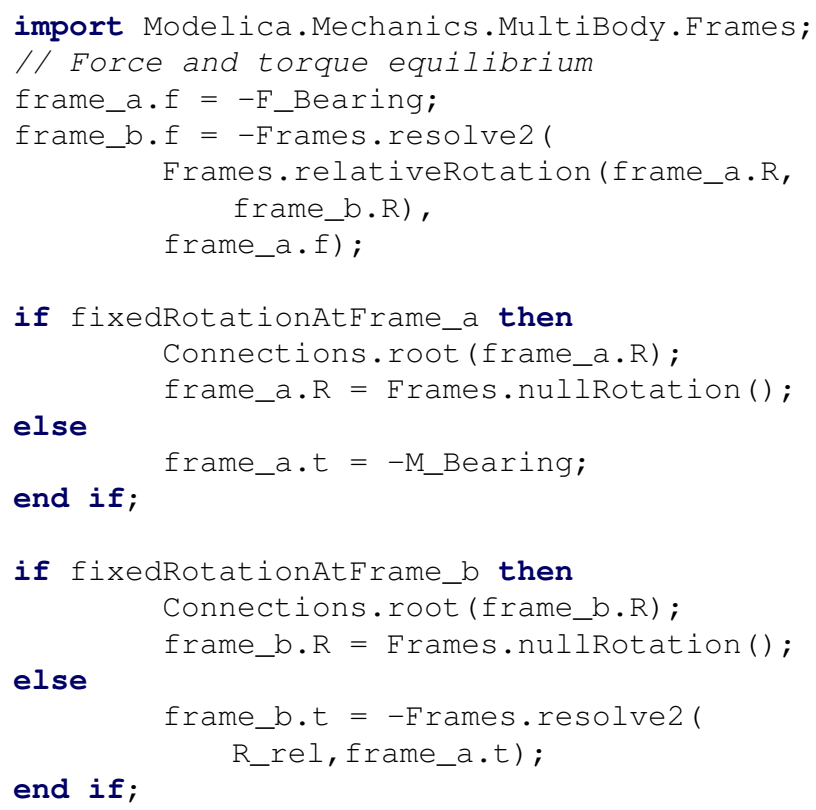

The calculation of the forces $F_{x b m}, F_{y b m}, F_{z b m}$ and torques $M_{x b m}, M_{y b m}, M_{z b m}$ on a bearing is realized in the sub-function calculateBearingForce(). The deformations $r \_r e l \_a, R_{-} r e l$ between the frame_a and frame_ $b$ of the bearing block are required for calculating bearing forces and torques. The angles $\beta_{x m}, \beta_{y m}, \varphi$ are calculated as consecutive rotations out of the rotation matrix $R_{-}$rel . The vector angles represents these angular deformations.

$$
\text { angles }=\left[\beta_{x m}, \beta_{y m}, \varphi\right]^{T}
$$

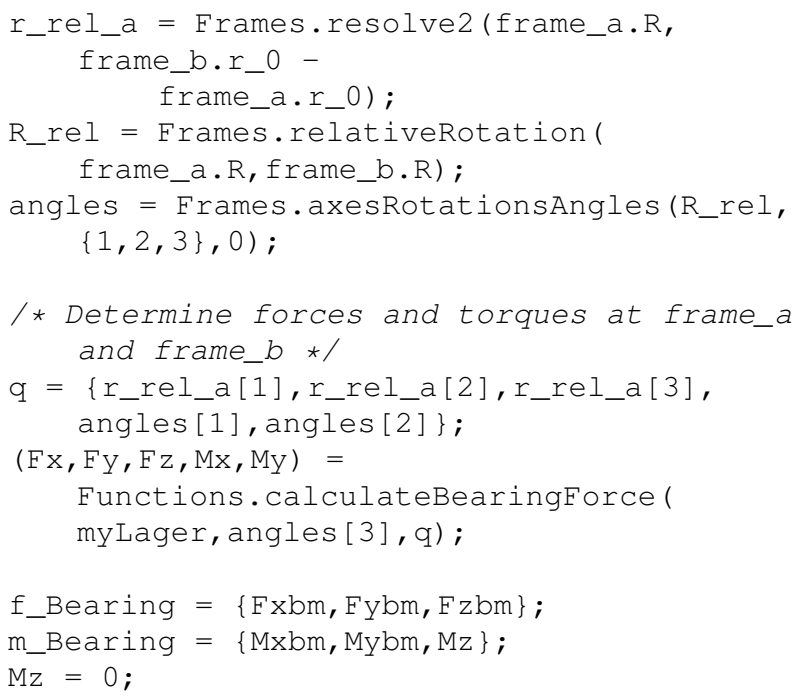

For the transformation of the forces and torques in the equations of motion the Jacobi matrix $\mathbf{J}$ is required. This matrix describes the kinematics on the bearing and is determined by means of the deformation $\mathbf{q}$. The Cartesian deformations $\delta_{i m}, i=x, y, z$ are modeled as sliders and the 
rotations $\beta_{i m}, i=x, y$ are modeled as consecutive rotation about $x, y$ with the rotation axis vector $\mathbf{z}_{i}$ using the rotation matrices $R_{x}, R_{y}$ (see Fig. 2).

$$
\begin{aligned}
\mathbf{z}_{i} & =\mathbf{e}_{x}, \mathbf{e}_{y} \\
\mathbf{J} & =\left[\begin{array}{l}
\mathbf{J}_{v i} \\
\mathbf{J}_{\omega i}
\end{array}\right] \\
\mathbf{J}_{i} & = \begin{cases}\mathbf{z}_{i} & , \text { translational motion } \\
\mathbf{z}_{i} \times \mathbf{p}_{i}^{*} & , \text { rotational motion }\end{cases} \\
\mathbf{J}_{\omega i} & = \begin{cases}\mathbf{0} & , \text { translational motion } \\
\mathbf{z}_{i} & , \text { rotational motion }\end{cases}
\end{aligned}
$$

The possible singularity of the Jacobi matrix for rotation about the $\mathrm{x}$ and $\mathrm{y}$ axes by $90^{\circ}$ is ignored since small deformations are expected.

The mass and inertia of the inner and outer ring of the bearing are modeled with the block "'Body"' of the MultiBody.Parts library. Each body is attached to frame_a and frame_ $b$ respectively. The dynamics of the rolling elements are neglected since only macroscopic effects of the system dynamics will be modeled.

Deriving a stiffness matrix $\mathbf{K}(\mathbf{q})$ is not required for the equations of motion in equation 26 . If required, the stiffness matrix $\mathbf{K}(\mathbf{q})$ can be calculated by means of the partial differentiation of the force and torque vectors $\mathbf{F}(\mathbf{q})$ according to the generalized coordinates of the bearing deformation $\mathbf{q}$ in equation 30 .

$$
\mathbf{K}(\mathbf{q})=\frac{\partial \mathbf{F}(\mathbf{q})}{\partial \mathbf{q}}
$$

\section{Simulation Results}

The parameters from table 1 are used to simulate the stiffness forces and torques with varying preload $F_{x m}$ and a partial rotation $\varphi=360 / Z$ of the bearing.

\begin{tabular}{|r|c|c|}
\hline Parameter & Value & Unit \\
\hline $\mathrm{R}$ & 34.45 & $\mathrm{~mm}$ \\
\hline $\mathrm{e}$ & 10 & $\mathrm{~mm}$ \\
\hline $\mathrm{Z}$ & 15 & \\
\hline$K_{n}$ & 395000 & $\mathrm{~N} / \mathrm{mm}^{\frac{3}{2}}$ \\
\hline$\alpha_{0}$ & 45 & \\
\hline$r_{L}$ & 0 & $\mathrm{~mm}$ \\
\hline$A_{0}$ & 0.52 & $\mathrm{~mm}$ \\
\hline$n$ & 1.5 & \\
\hline$\delta_{z, 0}$ & 0 & $\mathrm{~mm}$ \\
\hline
\end{tabular}

Table 1. Parameters of a double row roller bearing

Figure 6 shows a periodicity for the forces and torques within a bearing rotation. For each bearing rotation, the force and torque progression is repeated by the number of rolling elements. To assess the effects of parameter excitations on the system dynamics of the manipulator, further
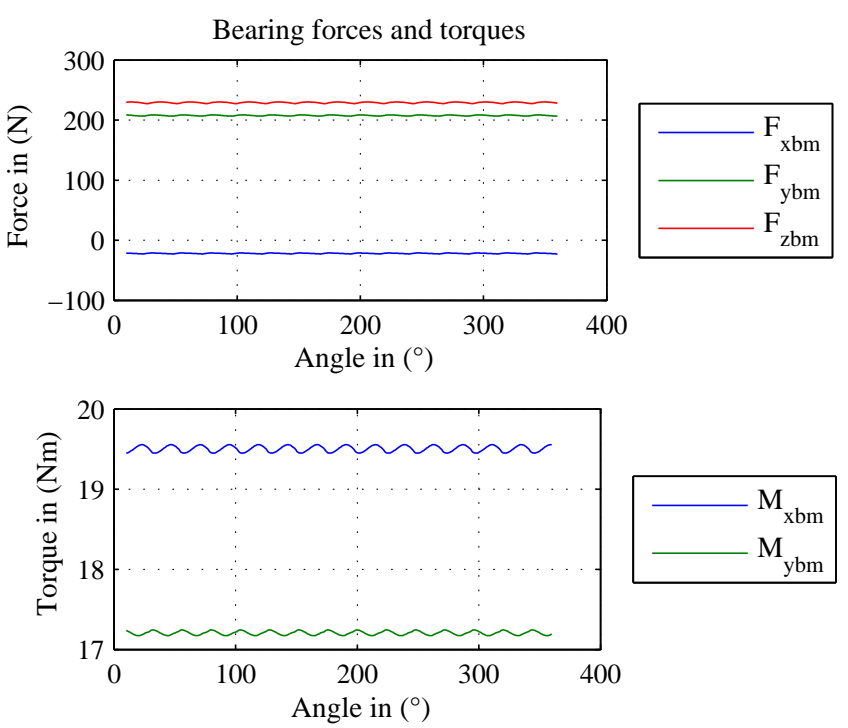

Figure 6. Bearing stiffness forces, bearing angle position varying

testing of the overall system of the manipulator is necessary. Next a simple example of a pendulum is shown. A stiff revolute joint and a bearing with its parameters in table 1 as a revolute joint are compared. The point mass is $1 \mathrm{~kg}$ and the length of the pendulum is $1 \mathrm{~m}$. Figure 7 shows the model in initial configuration. The reaction forces and torque in figure 8 . show the difference between the rigid and the elastic suspended joint of the pendulum.
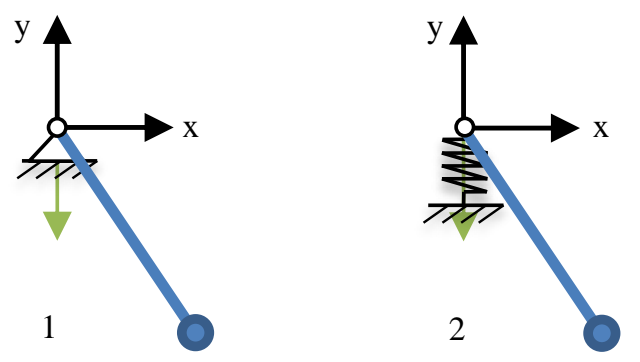

Figure 7. Pendulum, 1: rigid suspension 2: elastic suspension

\section{Conclusion and Outlook}

In this work the calculation of the stiffness forces and torques of single- and double-row bearings are shown. The following bearing types are considered:

- Single row ball bearing

- Single row roller bearing

- Double row ball bearing

- Double row roller bearing

- Cross roller bearing 

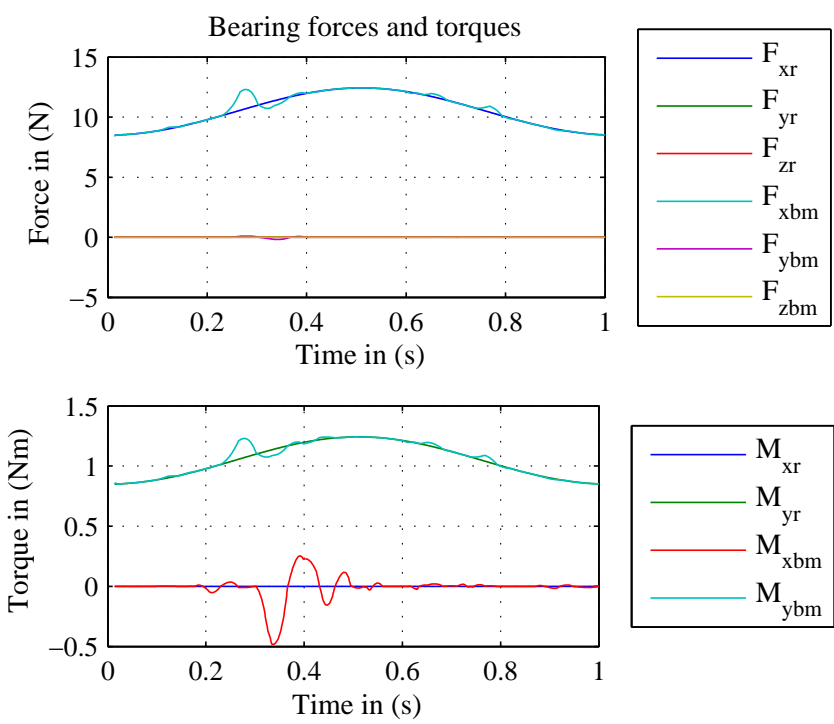

Figure 8. Forces and torques on the bearing and on the revolute joint and the displacement

The stiffness forces and torques are implemented in the MultiBody library of Modelica. A simulation shows the influence of loads and bearing position.

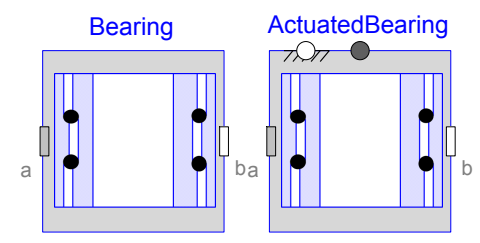

Figure 9. Bearing with $1 \mathrm{DOF}$ and actuated bearing

According to equation 12 and figure 2 the bearing model yields a free rotation about the Z-axis. For multibody systems and actuated mechanisms it will be important to consider the drivetrain. The scope of the future work is to develop an actuated bearing similar to the actuated joint in the Modelica MultiBody library. Further modeling and simulation of different mechanisms like a robot arm will be performed and validated.

\section{References}

Behnam Ghalamchi, Jussi Sopanen, and Aki Mikkola. Simple and versatile dynamic model of spherical roller bearing. International Journal of Rotating Machinery, 2013, 2013.

Aydin Gunduz. Multi-dimensional stiffness characteristics of double row angular contact ball bearings and their role in influencing vibration modes. $\mathrm{PhD}$ thesis, The Ohio State University, 2012.

Hans-Jürgen Hardtke, Bodo Heimann, and Heinz Sollmann. Lehr-und Übungsbuch Technische Mechanik Bd. II, Kinematik. Kinetik-Systemdynamik-Mechatronik, Fachbuchverlag Leipzig, 1997.

Teik C. Lim and Rajendra Singh. Vibration transmission through rolling element bearings, part i: bearing stiffness formulation. Journal of sound and vibration, 139(2):179-199, 1990a.

Teik C. Lim and Rajendra Singh. Vibration transmission through rolling element bearings, part ii: system studies. Journal of sound and vibration, 139(2):201-225, $1990 \mathrm{~b}$.

Wilhelm Matek, Dieter Muhs, Herbert Wittel, and Manfred Becker. Roloff/Matek Maschinenelemente: Normung Berechnung Gestaltung. Springer-Verlag, 2013.

Weihua Qian and Georg Jacobs. Dynamic simulation of cylindrical roller bearings. Technical report, Lehrstuhl und Institut für Maschinenelemente und Maschinengestaltung, RWTH Aachen, 2014.

Matej Tadina and Miha Boltežar. Improved model of a ball bearing for the simulation of vibration signals due to faults during run-up. Journal of sound and vibration, 330(17):4287-4301, 2011. 